\title{
Phthalate pollution and human exposures in plastic greenhouse vegetable production and consumption systems
}

\author{
Yanxia Zhang ${ }^{1}$, Biao Huang ${ }^{1 *}$, Xinkai Wang $^{l}$
}

${ }^{1}$ Key Laboratory of Soil Environment and Pollution Remediation, Institute of Soil Science, CAS, Nanjing 210008, China (* bhuang@issas.ac.cn)

Introduction: With the growth of amount of vegetables planted in plastic greenhouses consumption in China, the human exposure risk to phthalates has increased from plastic greenhouse production and consumption systems.

Objectives and Methods: We chose Shaanxi province in western China as the research site i) to measure phthalate accumulation in the components such as soil, vegetables, and air in plastic greenhouses by using GC-MSMS, ii) to evaluate phthalate exposure from vegetable intake, air inhalation, and other pathways in the system by the exposure scenario modeling method, iii) to test urinary phthalate metabolites for selected populations to quantify different exposure pathways.

Results and Discussion: DEHP and DBP were found to be the dominant compounds of phthalates in different components in the system. Phthalate concentration in soil of S. Shaanxi was significantly higher than that in middle area and lowest in N. area. Total phthalate in vegetables were 911$8607 \mu \mathrm{g} / \mathrm{kg} \mathrm{dw}$ with a median value of $3275 \mu \mathrm{g} / \mathrm{kg} \mathrm{dw}$. There was a significant positive correlation between the phthalate concentrations of vegetables and soils. Phthalates in air mainly exist as gaseous form, which is significantly higher level than that in outdoors.

Phthalate inhalation and vegetable intake are the main exposure pathways for the producers in plastic greenhouses. The shares of inhalation exposure and vegetable intake are over $50 \%$ and $35 \%$ of all exposure pathways from the system. DEHP and DnBP accounted for $31 \%$ and $43 \%$ for males and $20 \%$ and $45 \%$ for females to total phthalate exposure in the system, respectively. DEHP and DnBP metabolites caused by vegetable intake accounted for $4.5 \%$ and $14.2 \%$ of the total daily phthalate exposure for males and those accounted for $1.7 \%$ and $8.8 \%$ for females, respectively. Phthalate intake exposure level for urban adults was higher than that for rural adults.

Conclusions: The phthalate inhalation and vegetable intake exposure risks are significant, especially for greenhouse vegetable producers. It is necessary to adopt measures to control the risks. 